\title{
LINKS AND CUBIC 3-POLYTOPES
}

\author{
WEILING YANG AND FUJI ZHANG
}

\begin{abstract}
It is well known that a prime link diagram corresponds to a signed plane graph without cut vertices (Kauffman, 1989). In this paper, we present a new relation between prime links and cubic 3-polytopes. Let $S$ be the set of links such that each $L \in S$ has a diagram whose corresponding signed plane graph is the graph of a cubic 3-polytope. We show that all nontrivial prime links, except $(2, n)$-torus links and $(p, q, r)$-pretzel links, can be obtained from $S$ by using some operation of untwining. Furthermore, we define the generalized cubic 3-polytope chains and then show that any nontrivial link can be obtained from $\mathbb{S}$ by some untwining operations, where $\mathbb{S}$ is the set of links corresponding to generalized cubic 3-polytope chains. These results are used to simplify the computation of the Kauffman brackets of links so that the computing can be done in a unified way for many infinite families of links.
\end{abstract}

\section{INTRODUCTION}

It is well known that a link diagram corresponds to a signed plane graph. In [5], Kauffman showed that the Kauffman bracket polynomial of a link can be obtained by computing the generalized Tutte polynomial of its corresponding signed plane graph. In [7, Read and Whitehead showed the relation between a chain polynomial and a chromatic polynomial. Recently it was shown [10] that the Kauffman bracket polynomial of a signed plane graph can be obtained from its chain polynomial. Furthermore, an equivalent relation was defined on the set of link diagrams according to the homeomorphic types of their corresponding signed plane graphs, so that we can compute the Kauffman brackets for all the members of each equivalent class in a unified way. For prime links we only need to consider the signed plane graphs without cut vertices. If we neglect the signs, we can choose a graph without cut vertices or the vertices of degree two as the representatives for each equivalent class. Then the Kauffman bracket polynomial of members in each equivalent class can be easily obtained from the chain polynomial of their representative by a special parametrization. In [8, we call all these representatives universal graphs. In [10, the universal graphs (signs neglected) with cyclomatic number less than 5 were listed. The numbers of universal graphs with cyclomatic numbers 1, 2, 3 and 4 are 1, 2, 4 and 16, respectively. Examples were provided to compute the chain polynomials and their corresponding Kauffman bracket polynomials of the set of links in

Received by the editor October 23, 2006 and, in revised form, July 13, 2007.

2000 Mathematics Subject Classification. Primary 05C10; Secondary 57M25.

Key words and phrases. Link diagram, signed plane graph, cubic 3-polytope, generalized cubic 3-polytope chain, untwining, chain polynomial, Kauffman bracket polynomial.

The first author was supported in part by NSFC grant 10501038.

The second and corresponding author was supported in part by NSFC grant 10671162 .

(C)2008 American Mathematical Society Reverts to public domain 28 years from publication 
equivalent classes. It was also shown that there are 111 plane graphs without cut vertices or the vertices of degree two which are universal graphs (signs neglected) with cyclomatic number 5 . The 111 graphs were found in [4 by a computer search and proved in 9. Clearly it is a tedious task to compute the chain polynomials of these 111 graphs and use them to compute the Kauffman bracket polynomials of links. So, in [8, we reduced the universal graphs to be the 3-edge-connected plane graphs without cut vertices, so that the numbers of universal graphs with cyclomatic numbers $1,2,3,4$ and 5 are $1,1,3,10$ and 56, respectively (see 8), Figure 7.) We observed that some universal graphs can be obtained from others by contracting some edges, and so we further reduced the number of universal graphs with cyclomatic numbers 3,4 and 5 to 1,1 and 2, respectively.

In this paper we study the universal graphs more thoroughly. Let $S$ be the set of links such that each $L \in S$ has a diagram whose representative is the graph of a cubic 3 -polytope. We will show that all nontrivial prime links, except $(2, n)$-torus links and $(p, q, r)$-pretzel links, can be obtained from $S$ by using an operation of untwining. Thus we can reduce the universal graphs to be the graphs of cubic 3polytopes. Then the Kauffman bracket of a prime link except $(2, n)$-torus links and $(p, q, r)$-pretzel links can be easily computed by the chain polynomial of a signed plane graph in the reduced universal graph set by a special parametrization. In order to study general links, we define the generalized cubic 3-polytope chains and then show that any nontrivial link can be obtained from $\mathbb{S}$ by some untwining operations, where $\mathbb{S}$ is defined to be the set of links such that each $L \in \mathbb{S}$ has a diagram whose representative is a generalized cubic 3-polytope chain. These results are used to simplify the computation of the Kauffman brackets of links so that the computing can be done in a unified way for many infinite families of links. Moreover, we found the relation between the crossing number of a link and the cyclomatic number of its corresponding graph. With this relation we can find the Kauffman brackets of all links with small crossing numbers.

\section{Preliminaries}

A graph $G$ is an ordered triple $\left(V(G), E(G), \psi_{G}\right)$ consisting of a nonempty set $V(G)$ of vertices, a set $E(G)$, disjoint from $V(G)$, of edges, and an incidence function $\psi_{G}$ that associates each edge of $G$ with an unordered pair of (not necessarily distinct) vertices of $G$ (see [2] for the details). Throughout this paper, we use $d(u)$ to denote the degree of a vertex $u$, and use $\chi^{\prime}(G)$ to denote the edgeconnectivity of $G$. The cyclomatic number of a graph $G$, denoted by $n(G)$, equals $|E(G)|-|V(G)|+k(G)$, where $k(G)$ is the number of connected components of $G$.

The convex hull of a finite set of points $V$ in the Euclidean 3 -space is called the (convex) 3-polytope generated by $V$. The pair consisting of the set of vertices and the set of edges (1-faces) of a 3-polytope is called the graph of the 3-polytope.

Theorem 2.1 (Steinitz [3]). A simple graph is the graph of a 3-polytope if and only if it is planar and 3-connected.

A link $L$ with $n$ components is a subset of $\mathbb{R}^{3} \subset \mathbb{R}^{3} \cup\{\infty\}$, consisting of $n$ disjoint piecewise linear simple closed curves. A knot is a link of one component. Although links live in $\mathbb{R}^{3}$, we usually represent them by link diagrams: the regular projections of links into $\mathbb{R}^{2}$ with overpassing curves specified (see [6] for the details). 
Example 2.2. The diagrams of the (right-handed) trefoil knot and the Hopf link are shown in Figure 1.
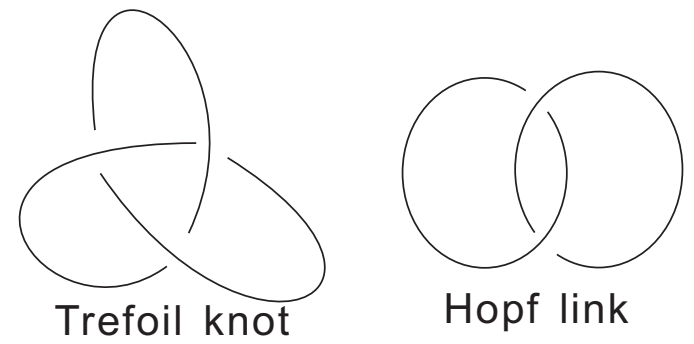

FiguRE 1. Link diagrams

A signed graph is a graph whose edges are each labeled with a sign $(+1$ or $-1)$. There is a correspondence between link diagrams and signed plane graphs via medial construction. Now we give a brief account of it (for details, see [5]).

Given a link diagram $D$, we first shade its faces checkerboardly. Then associate it with an edge-signed multigraph $G[D]$ as follows: for each shaded face $F$, take a vertex $v_{F}$ in $F$, and for each crossing at which two shaded faces $F_{i}$ and $F_{j}$ meet, take an edge $v_{F_{i}} v_{F_{j}}$. Furthermore, give each edge $v_{F_{i}} v_{F_{j}}$ a sign according to the type of the crossing as shown in Figure 2. We call the obtained graph a signed plane graph. Note that every link diagram has precisely two distinct shadings: one with the unbounded face shaded; the other with the unbounded face unshaded. So each link diagram corresponds to two signed plane graphs which are dual (see Figure 3.)

Conversely, for each signed plane graph $G$, we can construct the corresponding link diagram $D$. Firstly, we construct the medial graph $M(G)$ of the plane graph $G$ (without the signs). If $G$ is a connected nontrivial plane graph, $M(G)$ is a 4 regular plane graph defined as follows: its vertex set is obtained by taking a point on each edge of $G$; and two vertices are joined by an edge lying in a face of $G$ if they are on adjacent edges of the face so that each face $f$ of $G$ determines $r$ edges of $M(G)$ if the face $f$ has $r$ edges. If $G$ is trivial (that is, it is a single vertex), then $M(G)$ is a simple closed curve surrounding the vertex (strictly speaking, it is not a genuine graph). If $G$ is not connected, then $M(G)$ is the disjoint union of the medial graphs of its connected components. Secondly, we turn $M(G)$ into a link diagram $D=D(G)$ by converting each vertex of $M(G)$ into a crossing according to the sign of the edge where the vertex is located, as indicated in Figure 2.
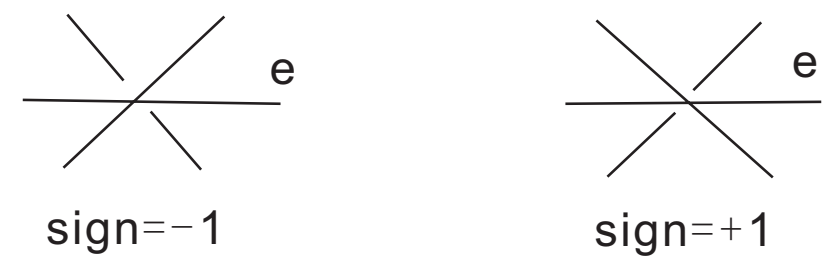

Figure 2. Signs of edges corresponding to two kinds of crossings 
Example 2.3. The diagram of knot $9_{44}$ and its two corresponding signed plane graphs are shown in Figure 3.
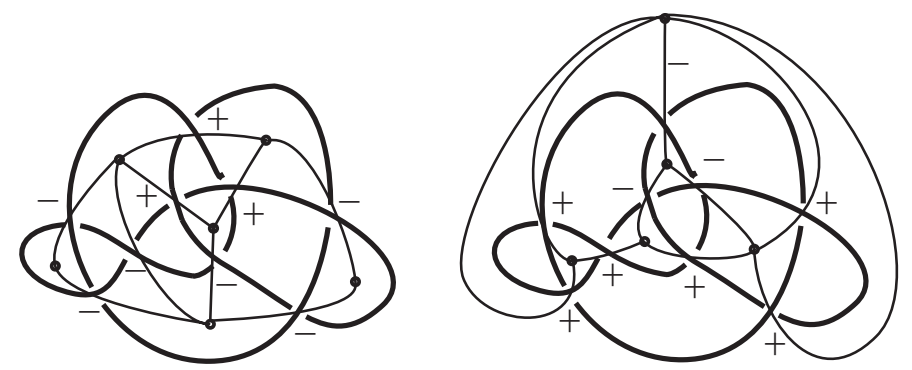

Figure 3. A link diagram (thick line) with two corresponding signed plane graphs (thin line)

Definition 2.4 ([5]). The Kauffman bracket polynomial $\langle D\rangle \in \mathbb{Z}\left[A, A^{-1}\right]$ of a link diagram $D$ is defined by the following two properties:

(1) The Kauffman bracket polynomial of a diagram consisting of $k$ disjoint simple closed curves in the plane is $d^{k-1}$, where $d=-A^{2}-A^{-2}$.

(2) $\langle D\rangle=A\left\langle D_{1}\right\rangle+A^{-1}\left\langle D_{2}\right\rangle$, where $D, D_{1}$ and $D_{2}$ are identical diagrams except that a crossing of $D$ is nullified in two different ways to form $D_{1}$ and $D_{2}$ respectively (see Figure 4).

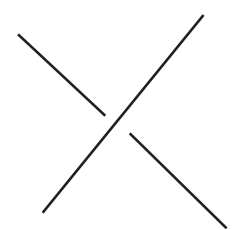

D

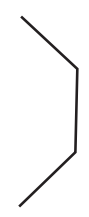

$\mathrm{D}_{1}$

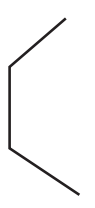

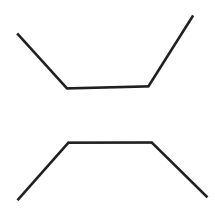

$\mathrm{D} 2$

FIGURE 4

Let $G=\left(V(G), E(G), \psi_{G}\right)$ be a graph, $\vec{G}=\left(V(G), \overrightarrow{E(G)}, \overrightarrow{\psi_{G}}\right)$ be an orientation of $G$. A $q$-flow is a map $f: \vec{E} \longrightarrow Z_{q}$ such that Kirchhoff's current law is satisfied, namely, for each vertex $v$ the total flow out of $v$ is equal to the total flow into $v$. A $q$-flow is said to be nowhere-zero if it has a nonzero value on each edge. One can easily see that the number of nowhere-zero $q$-flows does not depend on the orientation of $G$. So we write $F[G](q)$ for the number of nowhere-zero $q$-flows in $G$. It is not difficult to see that $F[G](q)$ is a polynomial in $q$ (see [1] for the details).

Definition 2.5 (7]). Let $M$ be a graph whose edges have been labeled with elements of a commutative ring with identity 1 . The chain polynomial $C h[M]$ of $M$ is defined as

$$
C h[M]=\sum_{Y} F[E(M-Y)](1-w) \varepsilon(Y)=\sum_{Y} F[Y](1-w) \varepsilon(E(M)-Y),
$$


where the summation is over all subsets of $E(M) ; F[Y]$ (respectively $F[E(M)-Y]$ ) is the flow polynomial of $\langle Y\rangle$ (respectively $\langle E(M)-Y\rangle$ ), the subgraph of $M$ induced by the edges in $Y$ (respectively $E(M)-Y) ; \varepsilon(Y)$ (respectively $\varepsilon(E(M)-Y)$ ) is the product of labels of the edges (we usually identify an edge with its label) in $Y$ (respectively $E(M)-Y$ ).

A path $P$ in a graph $M$ is called a chain in $M$ if every inner vertex of $P$ has degree 2 in the graph $M$ but the end vertices (not necessary distinct) of $P$ have degree not equal to 2 in $M$.

The following proposition is implicit in [7] and is presented in the current form in $[10$.

Proposition 2.6 ([10]). Let $a$ be a chain of $M$, and let $M-a$ and $M / a$ be the graphs obtained from $M$ by deleting and contracting the chain a respectively. Then if a is a loop, $C h[M]=(a-w) C h[M-a]$, and otherwise, $C h[M]=(a-1) C h[M-$ $a]+C h[M / a]$.

Definition 2.7 ([10]). A signed graph $G$ is special if it satisfies the property: for any chain of $G$, all edges of this chain have the same sign.

As pointed out in [10], every prime link has a corresponding special signed plane graph. To a special signed graph $G$, we define the length of a chain $a$ to be the sum of the signs of the edges in the chain, and denote it by $n_{a}$. It is not difficult to see that a special signed plane graph can be reduced by suppressing the vertices of degree 2 until suppression is no longer possible. We call this reduced graph the reduction of the signed plane graph, and denote it by $R(G)$. Obviously there is a one-to-one correspondence between the chains of $G$ and the edges of $R(G)$.

Theorem 2.8 ([10]). Let $D$ be a link diagram, $G$ be a special signed plane graph of $D$, and $R$ be the reduction of $G$. In $C h[R]$, if we replace $\omega$ by $-A^{4}-1-A^{-4}$, and replace $a$ by $\left(-A^{-4}\right)^{n_{a}}$ for every chain $a$, then

$$
\langle D\rangle=\frac{A^{m}}{\left(-A^{2}-A^{-2}\right)^{q-p+1}} C h[R],
$$

where $m$ is the sum of all signs of $G$, and $p$ and $q$ are the numbers of vertices and edges of graph $R$, respectively.

\section{MAin RESUlt}

In this section, we will show that, by using an operation, all nontrivial prime links, except $(2, n)$-torus links and $(p, q, r)$-pretzel links, can be obtained from $S$, and all nontrivial links can be obtained from $\mathbb{S}$, where $S$ is the set of links such that each $L \in S$ has a diagram whose corresponding signed plane graph is the graph of a cubic 3-polytope, and $\mathbb{S}$ is the set of links corresponding to generalized cubic 3-polytope chains (cf. Definition 3.5 below). Some examples are given to illustrate our approach.

Lemma 3.1. Let $M$ be a 3-edge-connected plane graph without cut vertices. If $M$ is not a cubic graph, then $M$ can be obtained from a 3-edge-connected cubic plane graph with the same cyclomatic number and without cut vertices by contracting some edges. 
Proof. Let $M_{0}=M$. We will proceed inductively to construct a finite sequence $\left(M_{0}, M_{1}, \ldots, M_{r}\right)$ of 3 -edge-connected graphs without cut vertices such that the number of vertices with degree more than 3 in $M_{i}$ is strictly decreasing when $i$ increases and the last graph $M_{r}$ in the sequence is cubic. Suppose $M_{0}, M_{1}, \ldots, M_{i}$ have been constructed where $M_{i}$ is not yet cubic. Arbitrarily take a vertex $u \in$ $V\left(M_{i}\right)$ with $d(u) \geqslant 4$. Then choose edges $e_{1}, e_{2}$ incident with $u$, so that there is no 3 -edge cut containing both $e_{1}$ and $e_{2}$. Then split the vertex $u d(u)-3$ times as illustrated in Figure 5, so that $u$ becomes a vertex of degree 3 .

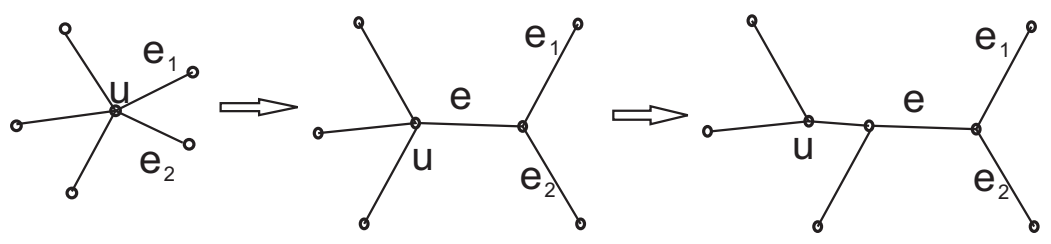

FIgURE 5. Splitting vertex $u$

Denote the resultant graph by $M_{i+1}$. Clearly, $M_{i+1}$ has the same cyclomatic number as $M_{i}$, and its number of vertices with degree more than 3 is less than that of $M_{i}$. Repeating this procedure we will finally get $M_{r}$ as a cubic graph.

To ensure that we can perform the operations of splitting and execute the inductive procedure to produce the sequence of 3-edge connected graphs $M_{0}, M_{1}, \ldots, M_{r}$ as desired, we only need to show the following two statements:

(S1) In $M_{i}$, if $d(u) \geqslant 4$, there exist two edges $e^{\prime}$ and $e^{\prime \prime}$ incident with $u$ such that for any edge $e \in E\left(M_{i}\right),\left\{e^{\prime}, e^{\prime \prime}, e\right\}$ is not an edge cut.

(S2) $M_{i+1}$ is a 3-edge-connected graph without cut vertices.

Proof of (S1): It's trivial if the edge-connectivity $\chi^{\prime}\left(M_{i}\right) \geq 4$. So we only consider the case $\chi^{\prime}\left(M_{i}\right)=3$. Assume that edges $e_{1}, e_{2}, e_{3}, e_{4}$ are incident with $u$. If for any edge $e \in E\left(M_{i}\right),\left\{e_{1}, e_{2}, e\right\}$ is not an edge cut, there is nothing to prove. So we assume that there exists an edge $e$ such that $\left\{e_{1}, e_{2}, e\right\}$ is an edge cut. Clearly, at least one of $e_{3}$ and $e_{4}$ is not $e$, say $e_{3} \neq e$. We claim that $\left\{e_{2}, e_{3}\right\}$ cannot be contained in any 3 -edge cut. Suppose, to the contrary, that there exists an edge $a$ such that $\left\{e_{2}, e_{3}, a\right\}$ is an edge cut. In the following we will show that a contradiction will occur. Noting that $M_{i}-\left\{e_{1}, e_{2}\right\}$ has a cut edge $e$, and $\chi^{\prime}\left(M_{i}\right)=3$, $M_{i}$ can be depicted as in Figure 6:

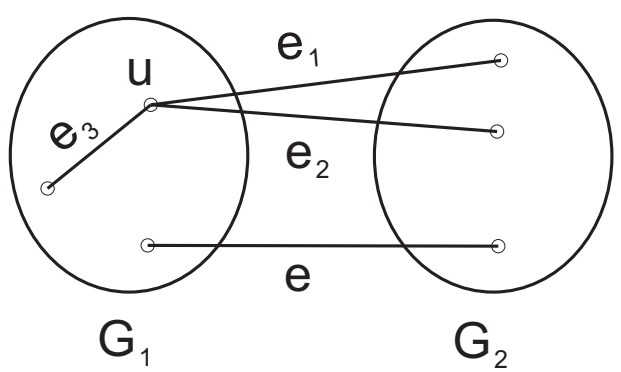

FiguRE $6 . M_{i}$ 
Clearly, $G_{1}$ and $G_{2}$ are both connected.

Case 1. $G_{1}$ and $G_{2}$ are not both 2-edge-connected. That is, for some $i \in\{1,2\}$, $G_{i}$ has a cut edge $b$. Then $G_{i}-b$ has exactly two components, say $H_{1}, H_{2}$. Let $\nabla\left(H_{j}\right)$ denote the set of edges joining the vertices of $H_{j}$ to the vertices in $V(G)-$ $V\left(H_{j}\right), j \in\{1,2\}$. Because $\nabla H_{1} \cup \nabla H_{2}=\left\{b, e_{1}, e_{2}, e\right\}$ and $\nabla H_{1} \cap \nabla H_{2}=\{b\}$, one of $\nabla H_{1}$ and $\nabla H_{2}$ is a 1- or 2-edge cut of $M_{i}$, which contradicts that $\chi^{\prime}\left(M_{i}\right)=3$.

Case 2. $G_{1}$ and $G_{2}$ are both 2-edge-connected. Now we will see the expected contradiction by considering the edge $a$.

- If $a=e$, then $G_{1}-\left\{e_{3}\right\}$ and $G_{2}$ are both connected. So $M_{i}-\left\{e_{2}, e_{3}, a\right\}$ is connected, a contradiction.

- If $a=e_{1}$, then $G_{1}-\left\{e_{3}\right\}$ and $G_{2}$ are both connected. So $M_{i}-\left\{e_{2}, e_{3}, a\right\}$ is connected, a contradiction.

- If $a \in E\left(G_{2}\right)$, then $G_{2}-\{a\}$ and $G_{1}-\left\{e_{3}\right\}$ are both connected. So $M_{i}-$ $\left\{e_{2}, e_{3}, a\right\}$ is connected, a contradiction.

- If $a \in E\left(G_{1}\right)$, we consider the following two cases:

subcase 1: $\left\{e_{3}, a\right\}$ is not an edge cut of $G_{1}$. Then $G_{1}-\left\{e_{3}, a\right\}$ is connected. So $M_{i}-\left\{e_{2}, e_{3}, a\right\}$ is connected, a contradiction.

subcase 2: $\left\{e_{3}, a\right\}$ is an edge cut of $G_{1}$. The graph can be illustrated as in Figure 7 , where $H_{1}$ and $H_{2}$ are connected since $G_{1}$ is 2-edge-connected.

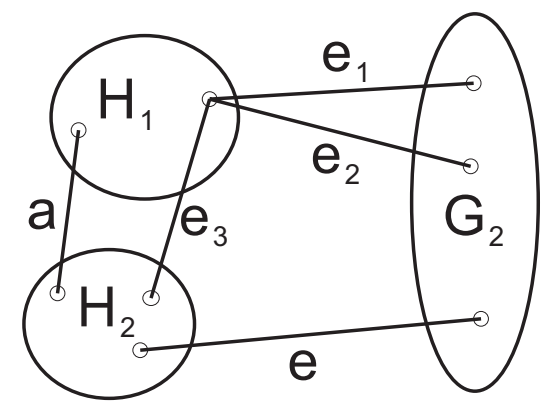

FiguRE $7 . M_{i}$

Note that one end of $e$ must be in $H_{2}$ (otherwise $\left\{a, e_{3}\right\}$ is a 2-edge cut of $M_{i}$, contradicting that $\left.\chi^{\prime}\left(M_{i}\right)=3\right)$. Thus $M_{i}-\left\{e_{2}, e_{3}, a\right\}$ is connected, a contradiction.

Proof of (S2): By contradiction. Suppose, to the contrary, that $\{a, b\}$ is an edge cut of $M_{i+1}$. Then one of $\{a, b\}$, say $a$, is the new edge resulting from a splitting (otherwise it contradicts that $\chi^{\prime}\left(M_{i}\right)=3$ ). From the process of splitting, we know that one end of $a$, say $u$, has degree 3. Suppose all the edges $e_{1}, e_{2}$, and $a$ are incident with $u$. Then $\left\{e_{1}, e_{2}, b\right\}$ is a 3 -edge cut of $M_{i}$. This contradicts the choice of $e_{1}, e_{2}$ (see Figure 8).

Clearly $M_{i+1}$ has no cut vertices since any vertex in $M_{i}$ is not a cut vertex. This completes the proof of (S2).

Thus we can inductively construct a desired finite sequence $\left(M_{0}, M_{1}, \ldots, M_{r}\right)$ of 3-edge-connected graphs without cut vertices such that the number of vertices with degree more than 3 in $M_{i}$ is strictly decreasing when $i$ increases and the sequence ends at a cubic graph $M_{r}$. When we reverse the process, we can get $M$ from $M_{r}$ by contracting some edges. 


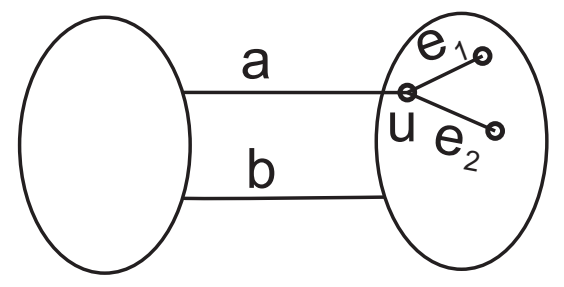

FiguRE 8. $M_{i+1}$

Lemma 3.2. Let $G$ be a 3-edge-connected cubic plane graph without cut vertices. Then $G$ is the graph of a cubic 3-polytope or the graph of two vertices joined by three multi-edges.

Proof. If $G$ is simple, then by an exercise in $\left[2, P_{44}, 3.1 .6\right], G$ is 3 -connected. By Theorem 2.1, $G$ is the graph of a cubic 3-polytope. If $G$ is not simple, it is clear that $G$ has no loops. There are only two cases to consider:

Case 1: $G$ has the local configuration shown in Figure 9.

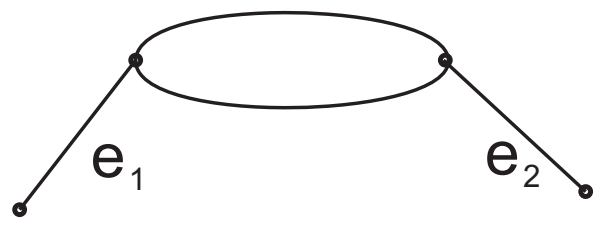

FiguRE 9. $G$

Then $\left\{e_{1}, e_{2}\right\}$ is a 2-edge cut, a contradiction.

Case 2: $G$ has two vertices joined by three multi-edges. Then, since $G$ is cubic, it has no more vertices or edges. So $G$ is the graph of two vertices joined by three multi-edges. The lemma is thus proved.

The untwining of links is the local transformation of some half twists to parallel strands as showing in Figure 10.
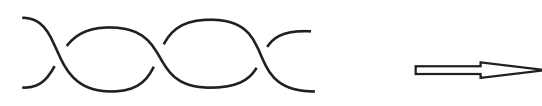

Figure 10. The operation of untwining

Theorem 3.3. Any nontrivial prime link, except $(2, n)$-torus links and $(p, q, r)$ pretzel links, can be obtained from a link in $S$ by some untwining operations, where $S$ is the set of links such that each $L \in S$ has a diagram whose corresponding signed plane graph is the graph of a cubic 3-polytope. Conversely, any graph $G$ of a cubic 3-polytope corresponds to some link diagrams with the minimal number of crossings. 
Proof. Given any nontrivial prime link $L$ that is neither a $(2, n)$-torus link nor a $(p, q, r)$-pretzel link, if it has a diagram whose corresponding reduced signed plane graph (signs neglected) is the graph of a cubic 3-polytope, then nothing needs to be proved. So, letting $D$ be a diagram of $L$, and $M$ be its corresponding reduced signed plane graph, we assume that $M$ is not the graph of a cubic 3-polytope. Since $M$ has no cut vertices, $M$ is 2-edge-connected. If $M$ is not 3-edge-connected and $L$ is not a $(2, n)$-torus link, then for each 2-edge cut of $M$, we writhe $D n_{b}$ times to eliminate it as shown in Figure 11. Then we get a diagram $D^{\prime}$ of the same link $L$, and the reduced signed plane graph of $D^{\prime}$ is 3-edge-connected (for details, see [8], theorem 2).

Without loss of generality we may assume that $M$ is a 3-edge-connected plane graph. By Lemma 3.1, $M$ can be obtained from a 3-edge-connected cubic plane graph $R$ by contracting some edges. Since $L$ is neither a $(2, n)$-torus link nor a $(p, q, r)$-pretzel link, by Lemma 3.2, $R$ is the graph of a cubic 3-polytope. One can see that the effect of the untwining operation to the link corresponds to that of the contraction operation to its corresponding graph (see Figure 12). So $L$ can be obtained from a link in $S$ by some untwining operations.
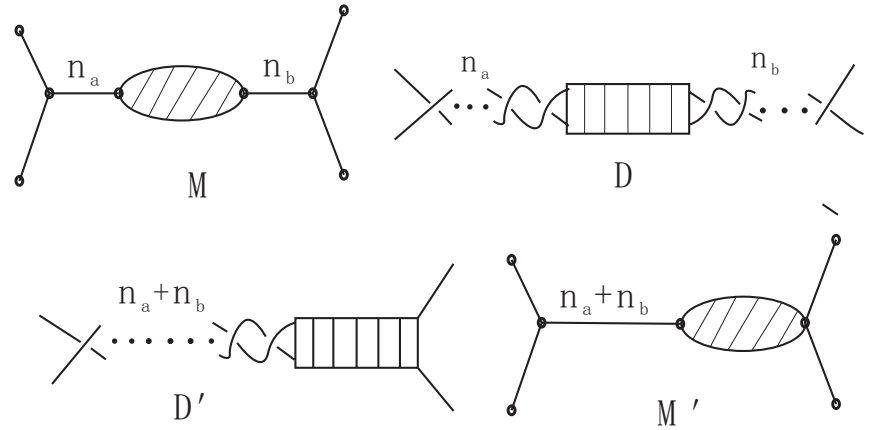

FiguRE 11. Get $M^{\prime}$ from $M$, where $D^{\prime}$ is another diagram of $L$

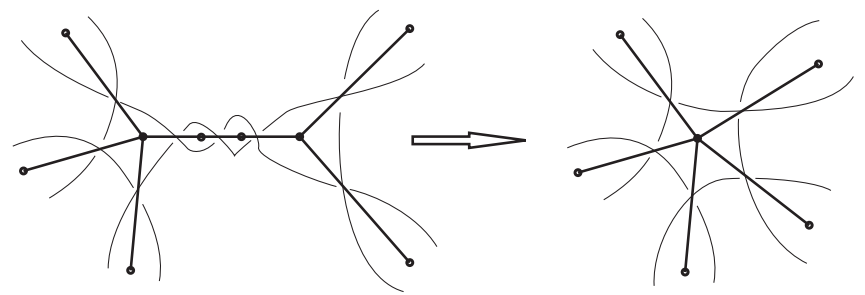

FIGURE 12. The effect of the untwining operation

For any graph of a cubic 3-polytope, if we give every edge a sign +1 , then we get an alternative link diagram $L$. Clearly there are no nugatory crossings in $L$. Note that a crossing is said to be a nugatory crossing if some two of the local regions appearing at the crossing are parts of the same region in the whole diagram. A nugatory crossing appears as a loop or a cut edge in the signed plane graph, which 
cannot exist in any graph of a cubic 3-polytope. By Theorem 22 in [1, p. 371] and the remark after it, we know that the alternative link diagram $L$ has the minimal number of crossings. The theorem is thus proved.

Remark 3.4. Theorem 3.3 claims that any nontrivial prime link $L$, except $(2, n)$ torus links and $(p, q, r)$-pretzel links, can be produced from a link in $S$, and so the signed plane graph of $L$ can be obtained from the graph $M$ of a cubic 3-polytope. However, we need to point out that $M$ is not unique. An example is shown in Figure 13.
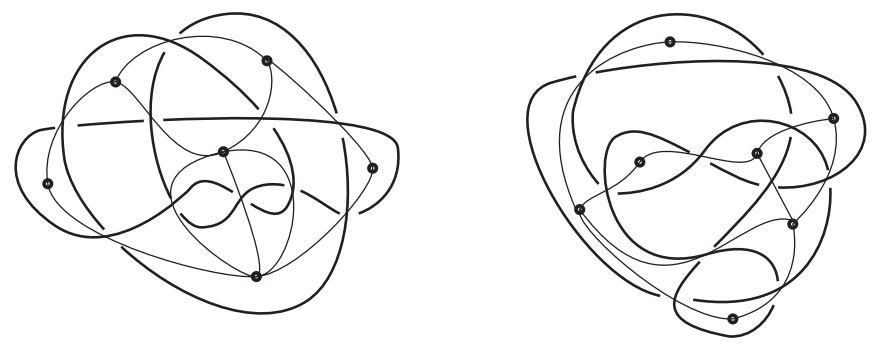

Figure 13. Perko pair knots (thick line) and their signed plane graphs (thin line)

It is well known that the Perko pair knots are the same knot. However, we can see that one of the Perko pair knots can have its reduced signed plane graph obtained from $R_{1}^{5}$ (see Figure 17) by contracting the edges $e, d, g, h$, and the other of the Perko pair knots can have its reduced signed plane graph obtained from $R_{1}^{4}$ (see Figure 17) by contracting $c, d$.

Now we give three examples in Figure 14 to illustrate how to construct an alternative link diagram with the minimum number of crossings from the graph of a cubic 3-polytope. From the diagrams, we know that these three links are all periodic links with periods 3,4 and 5, respectively.

As for the other links corresponding to platonic solids, octahedron and icosahedron are mirror images of the cube and the dodecahedron, respectively. Now we consider the general nontrivial links that do not need to be prime.

Definition 3.5. A generalized cubic 3-polytope chain is the graph which can be depicted as a "path" of graphs (see Figure 15), where $G_{i}$ is the graph of a cubic 3polytope, or a single vertex with a loop, or two vertices joined by three multi-edges, and each $G_{i}$ has only a single vertex in common with the next.

Theorem 3.6. Any nontrivial link can be obtained from a link in $\mathbb{S}$ by some untwining operations, where $\mathbb{S}$ is the set of links such that each $L \in \mathbb{S}$ has a diagram whose corresponding signed plane graph is the graph of a generalized cubic 3-polytope chain.

Proof. For any nontrivial link $K$, there exist nontrivial prime links $K_{1}, K_{2}, \ldots, K_{j}$ $(j \geqslant 1)$ such that $K=K_{1} \# K_{2} \# \ldots \# K_{j}$. Precisely, any link can be factored into a finite number of prime links, and these prime factors are uniquely determined up to order. Based on the relation between the operation of untwining to link and the operation of contraction to its corresponding signed plane graph, we will show 


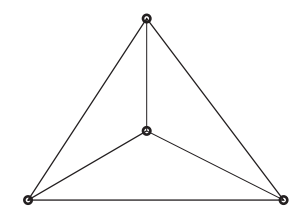

Tetrahedron

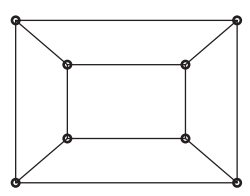

Cube

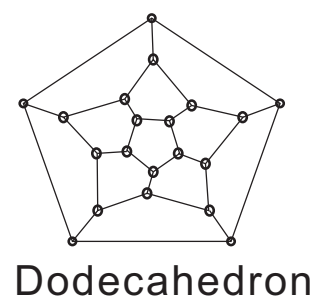

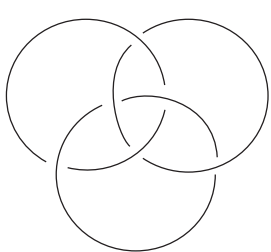
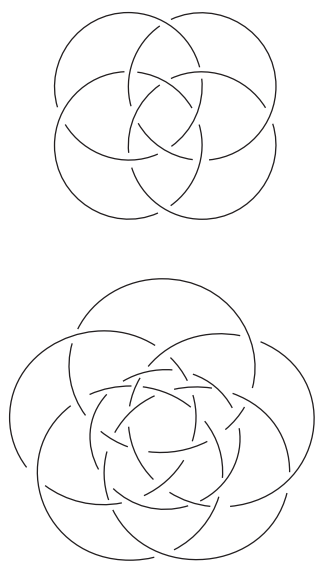

Figure 14. Three graphs of cubic 3-polytopes and their corresponding link diagrams

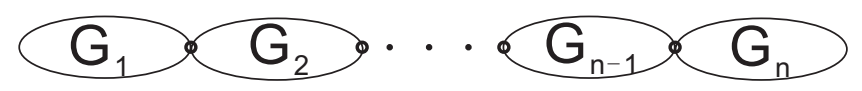

FIGURE 15. A generalized cubic 3-polytope chain

that there exists a reduced signed plane graph of $K$ which can be obtained from a generalized cubic 3-polytope chain by contracting some edges. Now we identify the link with its diagram. Suppose $G_{i}(1 \leqslant i \leqslant j)$ is the reduced signed plane graph corresponding to $K_{i}$. If $j=1$, by Theorem $3.3, G_{1}$ can be obtained from the graph of a cubic 3-polytope by contracting some edges, provided $K=K_{1}$ is neither a $(2, n)$-torus link nor a $(p, q, r)$-pretzel link. Note that the reduced signed plane graphs of $(2, n)$-torus links and $(p, q, r)$-pretzel links are a single vertex with a loop, and two vertices joined by three multi-edges, respectively. So the reduced signed plane graph $G_{1}$ of $K$ can be obtained from a generalized cubic 3-polytope chain by contracting some edges. If $j=2$ we make $K_{1} \# K_{2}$ as follows: one of the shaded regions $\gamma_{1}$ in $K_{1}$ is joined to one of the shaded regions $\gamma_{2}$ in $K_{2}$. Suppose that $u_{1} \in K_{1}$ and $u_{2} \in K_{2}$ correspond to $\gamma_{1}, \gamma_{2}$ in the signed plane graphs of $K_{1}$ and $K_{2}$, respectively. Then the graph obtained by identifying $u_{1}$ and $u_{2}$ is the signed plane graph of $K_{1} \# K_{2}$. Note that in $K_{1} \# K_{2}, K_{1}\left(K_{2}\right)$ can slide along the strand of $K_{2}\left(K_{1}\right)$. So the resultant $K_{1} \# K_{2}$ does not depend on the choice of the regions $\gamma_{1}, \gamma_{2}$. Thus we can choose the regions such that $d\left(u_{1}\right), d\left(u_{2}\right) \geqslant 3$, provided neither $G_{1}$ nor $G_{2}$ is a single vertex with a loop. Now we can conclude that $H=G_{1} \cup G_{2}$ 
with $G_{1}$ and $G_{2}$ sharing one vertex (see Figure 16) is the reduced signed plane graph of $K_{1} \# K_{2}$.

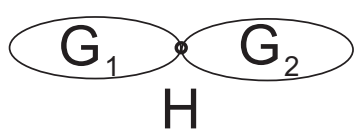

FiguRE 16. $H$

By Theorem 3.3, $G_{i}(i=1,2)$ can be obtained from the graph of a cubic 3polytope by contracting some edges, or $G_{i}$ is a single vertex with a loop, or two vertices joined by three multi-edges. So the reduced signed plane graph $H$ of $K_{1} \# K_{2}$ can be obtained from a generalized cubic 3-polytope chain by contracting some edges. By induction on $j$, we have proved the corollary.

From Theorem 3.3, we know that the graphs of cubic 3-polytopes are the core of link diagrams. It is known that the numbers of graphs of cubic 3-polytopes with cyclomatic numbers 3, 4, 5 and 6 are 1, 1, 2 and 5, respectively (see Figure 17). Usually we list the links according to their crossing numbers. On the other hand, we can list the cubic 3-polytopes according to their cyclomatic numbers. A natural problem is to consider the relation between the crossing number of a link and the cyclomatic number of its corresponding signed plane graphs. Clearly the results can be used to exhaust the links with small crossing numbers.

In section 2, we have pointed out that a link diagram corresponds to a pair of dual signed plane graphs. Furthermore, we will have following:

Theorem 3.7. For any link diagram $D$, at least one of its dual signed plane graphs has cyclomatic number at most $\left\lfloor\frac{n}{2}\right\rfloor$, where $n$ is the crossing number of $D$.

Proof. We may view the link diagram $D$ as a 4-regular plane graph $H$ whose vertices are the crossings of $D$. So $|V(H)|=n,|E(H)|=2 n$. By Euler's formula, $|V(H)|-$ $|E(H)|+|\phi(H)|=2$, where $|\phi(H)|$ is the number of faces of $H$. So $|\phi(H)|=n+2$.

Let $G_{1}, G_{2}$ be the dual signed plane graphs corresponding to $D$. Then $\left|E\left(G_{1}\right)\right|=$ $\left|E\left(G_{2}\right)\right|=n,\left|V\left(G_{1}\right)\right|+\left|V\left(G_{2}\right)\right|=|\phi(H)|=n+2$. Suppose $\left|V\left(G_{1}\right)\right| \geqslant\left|V\left(G_{2}\right)\right|$. Then $G_{1}$ has the cyclomatic number $n\left(G_{1}\right)=\left|E\left(G_{1}\right)\right|-\left|V\left(G_{1}\right)\right|+1 \leqslant n-\left\lceil\frac{n+2}{2}\right\rceil+$ $1=\left\lfloor\frac{n}{2}\right\rfloor$.

By Theorem 3.7, if we know the chain polynomials of the graphs of cubic 3polytopes with cyclomatic numbers no more than $n$, we can compute the Kauffman bracket polynomials of prime links with crossing numbers no more than $2 n+1$.

\section{Computing of Chain polynomial and Kauffman BRaCKet polynomial}

By Theorem 3.3, we know that any nontrivial prime link diagram $D$, except the diagrams of $(2, n)$-torus links and $(p, q, r)$-pretzel links, can be obtained by contracting some edges of the corresponding graph $M$ of a cubic 3-polytope. In other words, we proved that the set of universal graphs for prime links consists of the graphs of cubic 3-polytopes, besides two special cases which are a loop and two vertices with three multi-edges. For general links, the corresponding universal graphs are the generalized cubic 3-polytope chains. By the properties of a chain polynomial, it can be seen that when we use 1 to replace each contracted edge $a$ 
that appears in $C h[M]$, we get $C h[D]$. Then by Theorem 2.8, we can compute the Kauffman bracket polynomial of $D$. So the Kauffman bracket polynomial of any nontrivial link, except $(2, n)$-torus links and $(p, q, r)$-pretzel links, can be computed from the chain polynomial of the signed plane graph of a cubic 3-polytope by a special parametrization. On the other hand, the chain polynomials of the signed plane graphs of $(2, n)$-torus links and $(p, q, r)$-pretzel links have been found in 8$]$, and so their Kauffman bracket polynomials can be obtained easily.

By Theorem 3.7, if we know the chain polynomials of the graphs of all cubic 3polytopes with cyclomatic number no more than 6 , we can compute the Kauffman bracket polynomials of all prime links with crossing number no more than 13 . The chain polynomials of graphs of cubic 3-polytopes with cyclomatic number no more than 5 have been listed in 8 . In Figure 17 we list all the graphs of cubic 3-polytopes with cyclomatic number no more than 6 . The chain polynomials of graphs of cubic 3 -polytopes with cyclomatic number 6 have been obtained by using the computer algebra system MAPLE and are presented in [12].

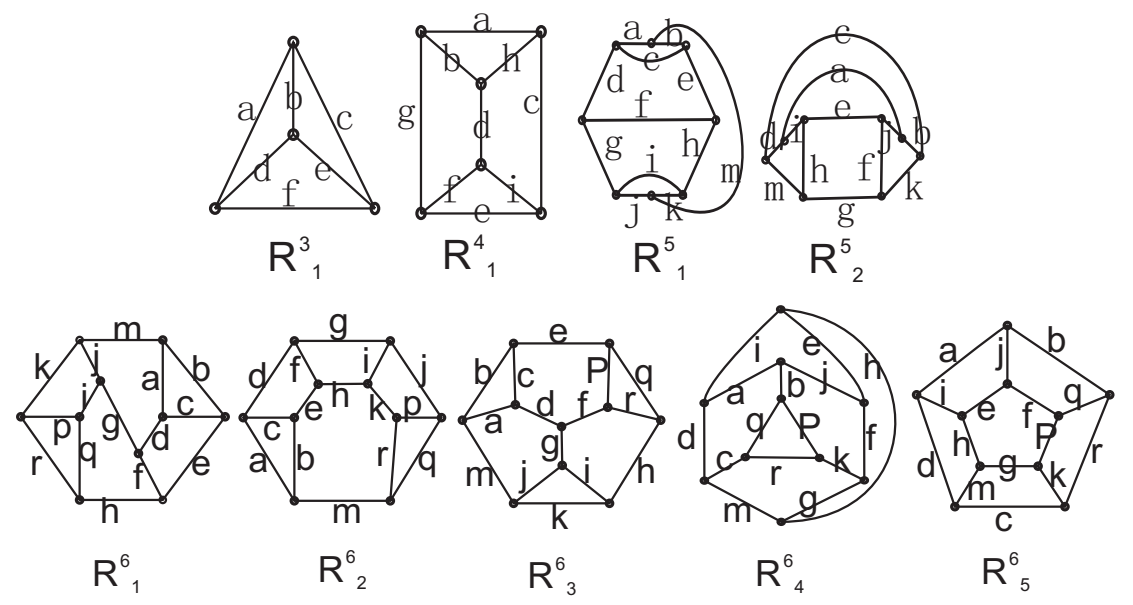

FiguRE 17. The graphs of cubic 3-polytopes with cyclomatic numbers $3,4,5$ and 6

By observation, we find that the chain polynomials of graphs of cubic 3-polytopes can be obtained by the chain polynomials of some graphs of cubic 3-polytopes with smaller cyclomatic numbers. For example, by Proposition 2.6 we can get the chain polynomial of $R_{2}^{6}$ as shown in Figure 18.

If we have known $C h\left[R_{1}^{5}\right]$, by making a proper change of labels, we can get $C h[G]$ and $C h[H]$. Then we get $C h\left[R_{2}^{6}\right]$. Obviously this approach can allow us to go further.

Remark 4.1. It has been pointed out in [7] that a list of 3-edge-connected cubic graphs would be sufficient to give us the chain polynomials for all graphs with a given value of $|E(G)|-|V(G)|$. But there is a gap in the proof. Every graph $M_{1}$ can be obtained from a cubic graph $M_{2}$, but $M_{2}$ need not be 3-edge-connected. If we do the operation in [7. Corollary of Theorem 4(b)] to get a 3-edge-connected graph $M_{3}, M_{3}$ may not be a cubic graph (see Figure 19). But this gap can be fixed by using Lemma 3.1 . 

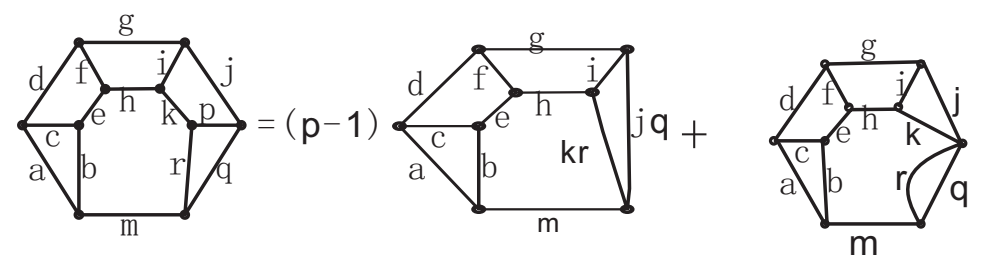

$\mathrm{R}_{2}^{6}$

$=(p-1)$
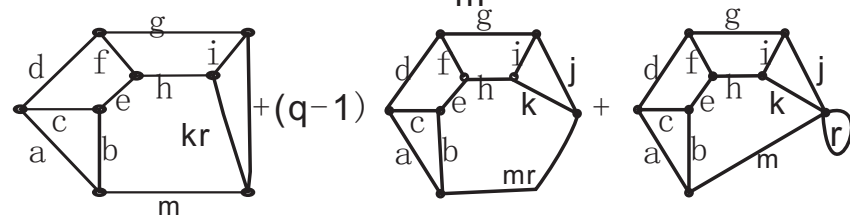

$=(p-1) \operatorname{Ch}[G]+(q-1) \operatorname{Ch}[H]+(r-w) \operatorname{Ch}\left[R^{5}{ }_{1}\right]$

FIGURE 18

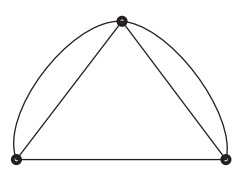

$\mathrm{M}_{1}$

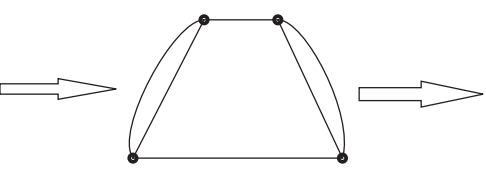

$\mathrm{M}_{2}$

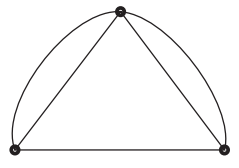

$\mathrm{M}_{3}$

FiguRE 19

Example 4.2 (see Figure 20). Compute the Kauffman bracket polynomial of the knot $13_{9221}$ by using the chain polynomial of $R_{3}^{6}$ and a special parametrization.
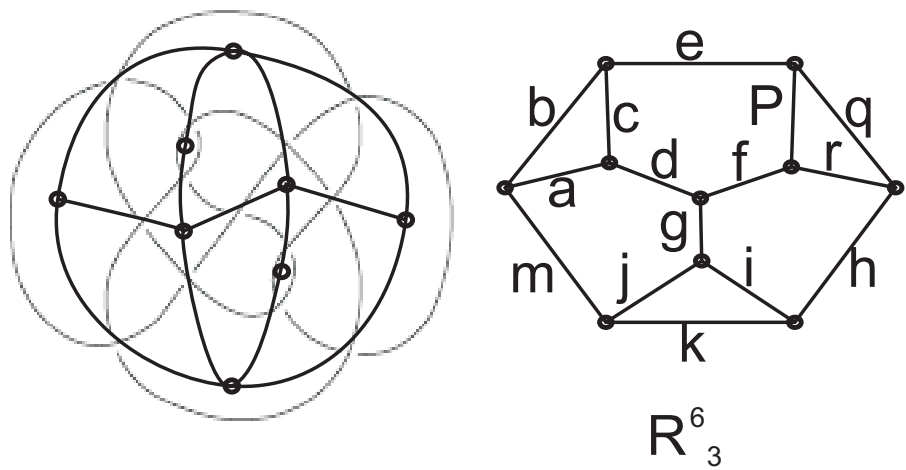

FiguRE 20. The corresponding signed plane graph of knot $13_{9221}$, where $e=g=h=m=1, n_{b}=n_{i}=2, n_{a}=n_{c}=n_{d}=n_{f}=$ $n_{j}=n_{k}=n_{p}=n_{q}=n_{r}=-1$.

First, we let $e=g=h=m=1$ in $C h\left[R_{3}^{6}\right]$. Second, we replace $w$ by $-A^{4}-$ $1-A^{-4}$, replace each of the chains $a, c, d, f, j, k, p, q, r$ by $-A^{4}$, and replace each of 
the chains $b, i$ by $\left(-A^{-4}\right)^{2}$. Then we get the chain polynomial of the signed plane graph of $13_{9221}$. By Theorem 2.8, we have:

$$
\begin{aligned}
\langle D\rangle= & \frac{A^{-5}}{\left(-A^{2}-A^{-2}\right)^{6}} \operatorname{Ch}\left[R_{3}^{6}\right] \\
= & A^{-25}-5 A^{-21}+10 A^{-17}-15 A^{-13}+19 A^{-9}-20 A^{-5}+19 A^{-1} \\
& -15 A^{3}+10 A^{7}-5 A^{11} .
\end{aligned}
$$

Example 4.3 (see Figure 21). Compute the Kauffman bracket polynomial of the knot $15_{203432}$ by using the chain polynomial of $R_{5}^{6}$ and a special parametrization.

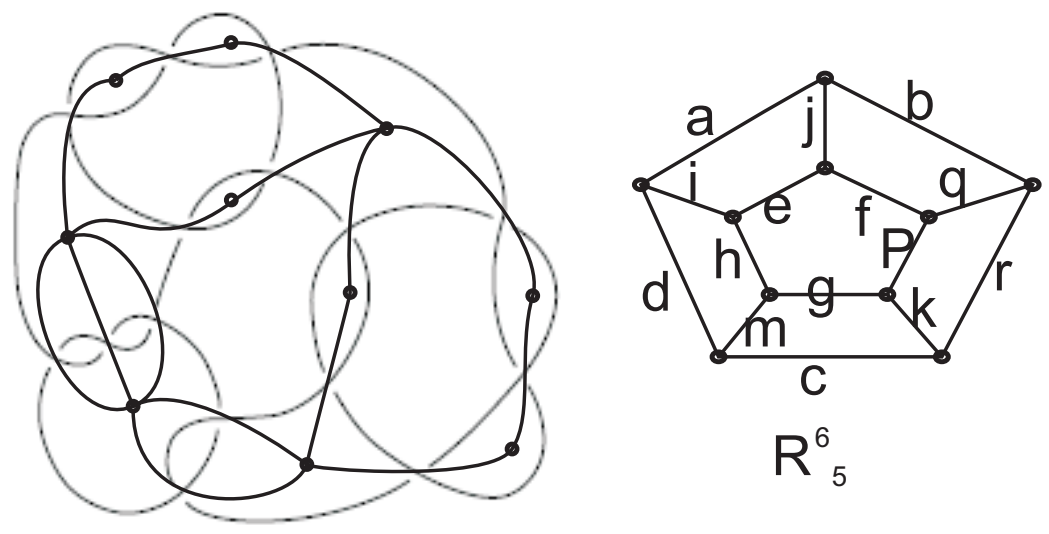

Figure 21. The corresponding signed plane graph of knot $15_{203432}$, where $a=b=c=e=f=g=1, n_{r}=n_{k}=2, n_{d}=$ $n_{m}=3, n_{h}=n_{i}=n_{j}=n_{p}=n_{q}=-1$.

First, we let $a=b=c=e=f=g=1$ in $C h\left[R_{5}^{6}\right]$. Second, we replace $w$ by $-A^{4}-1-A^{-4}$, replace each of the chains $h, i, j, p, q$ by $-A^{4}$, replace each of the chains $r, k$ by $\left(-A^{-4}\right)^{2}$, and replace each of the chains $d, m$ by $\left(-A^{-4}\right)^{3}$. Then we get the chain polynomial of the signed plane graph of $15_{203432}$. By Theorem 2.8 , we have:

$$
\begin{aligned}
\langle D\rangle= & \frac{A^{5}}{\left(-A^{2}-A^{-2}\right)^{6}} \operatorname{Ch}\left[R_{5}^{6}\right] \\
= & A^{-35}-4 A^{-31}+10 A^{-27}-20 A^{-23}+29 A^{-19}-36 A^{-15}+39 A^{-11} \\
& -35 A^{-7}+30 A^{-3}-19 A+10 A^{5}-4 A^{9}-A^{13}-A^{21} .
\end{aligned}
$$

Example 4.4 (see Figure 22). Compute the Kauffman bracket polynomial of the knot $15_{203969}$ by using the chain polynomial of $R_{5}^{6}$ and a special parametrization.

Firstly, we let $a=b=c=e=f=g=1$ in $C h\left[R_{5}^{6}\right]$. Secondly, we replace $w$ by $-A^{4}-1-A^{-4}$, replace each of the chains $i, j, k, m, q$ by $-A^{4}$, replace each of $r, p$ by $\left(-A^{-4}\right)^{2}$, and replace each of $d, h$ by $\left(-A^{-4}\right)^{3}$. Then we get the chain polynomial 

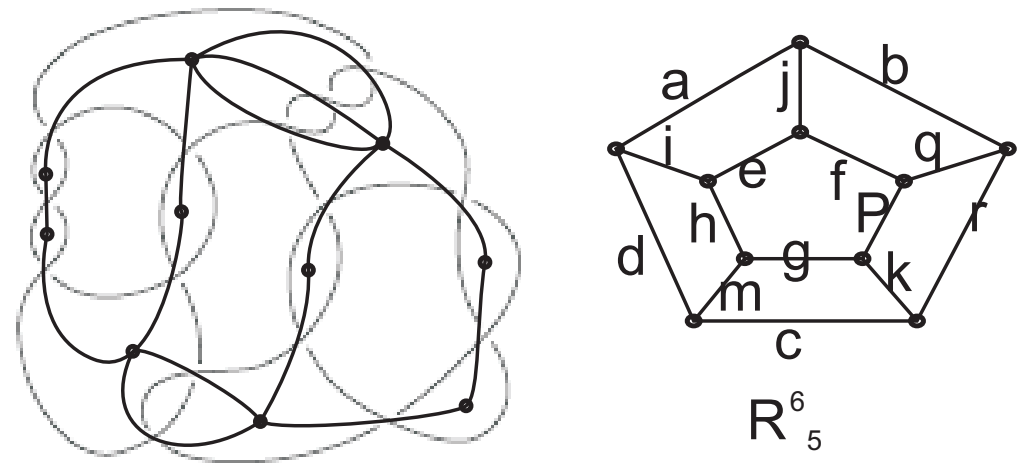

Figure 22. The corresponding signed plane graph of knot $15_{203969}$, where $a=b=c=e=f=g=1, n_{r}=n_{p}=2, n_{d}=$ $n_{h}=3, n_{i}=n_{j}=n_{k}=n_{m}=n_{q}=-1$.

of the signed plane graph of $15_{203969}$. By Theorem 2.8, we have:

$$
\begin{aligned}
\langle D\rangle= & \frac{A^{5}}{\left(-A^{2}-A^{-2}\right)^{6}} C h\left[R_{5}^{6}\right] \\
= & A^{-35}-4 A^{-31}+10 A^{-27}-20 A^{-23}+29 A^{-19}-36 A^{-15}+39 A^{-11} \\
& -35 A^{-7}+30 A^{-3}-19 A+10 A^{5}-4 A^{9}-A^{13}-A^{21} .
\end{aligned}
$$

Finally, we would like to give two remarks.

Remark 4.5. As pointed out in [10], for each universal graph, we can compute the Kauffman brackets of an infinite family of links by special parametrization. From the table of links, we observe that for most links, their corresponding signed plane graphs have vertices of degree two. So our method can simplify the computing procedure.

Remark 4.6. In [11, Read developed techniques by some graph operations to compute the chain polynomials of graphs. His result can also be used to compute the chain polynomials here. But with the aid of MAPLE, our method with parametrization is more straightforward.

\section{ACKNOWLEDGEMENT}

We would like to thank professor Zhibo Chen and Dr. Xian'an Jin for their helpful comments.

\section{REFERENCES}

1. B. Bollobas, Modern Graph Theory, Springer-Verlag, New York, 1998. MR,1633290 (99h:05001)

2. J.A. Bondy and U.S.R. Murty, Graph Theory with Applications, North-Holland, Amsterdam. 1981.

3. A. Brøndsted, An Introduction to Convex Polytopes, Springer-Verlag, New York, 1982. MR683612 (84d:52009)

4. B.R. Heap, The enumeration of homeomorphically irreducible star graphs, Journal of Mathematical Physics, 7 (1966), 1852-1857. MR0202638 (34:2500) 
5. L.H. Kauffman, A Tutte polynomial for signed graphs, Discrete Appl. Math. 25 (1989), 105127. MR.1031266 (91c:05082)

6. L.H. Kauffman, Knots and Physics, third edition, World Scientific Publishing Co., River Edge, NJ, 2001. MR1858113 (2002h:57012)

7. R.C. Read and E.G. Whitehead, Chromatic polynomials of homeomorphism classes of graphs, Discrete Math. 204 (1999), 337-356. MR1691877 (2000b:05059)

8. Weiling Yang and Fuji Zhang, The Kauffman bracket polynomial of links and universal signed plane graph, Lecture Notes in Computer Science 4381, 228-244, Springer-Verlag, New York, 2007.

9. Weiling Yang and Xian'an Jin, The construction of 2-connected plane graph with cyclomatic number 5, (in Chinese) Journal of Mathematical Study, 37 (2004), 83-95. MR 2229412

10. Xian'an Jin and Fuji Zhang, The Kauffman brackets for equivalence classes of links, Advances in Appl. Math. 34 (2005), 47-64. MR2102274 (2005j:57009)

11. R.C. Read, Chain polynomials of graphs, Discrete Math. 265 (2003), 213-235. MR.1969375 (2004c:05074)

12. http://math.xmu.edu.cn/school/teacher/fzzhang/fuji_zhang.html

School of Mathematical Sciences, Xiamen University, Xiamen, Fujian 361005, PeoPLE's RePublic OF China

E-mail address: ywlxmu@163.com

School of Mathematical Sciences, Xiamen University, Xiamen, Fujian 361005, PeoPLE'S RePublic OF China

E-mail address: fjzhang@xmu.edu.cn 